\section{SQUID-magnetometer with open-ended horizontal room-temperature access}

\author{
H J M ter Brake, J A Ulfman and J Flokstra \\ Twente University of Technology, PO Box 217, 7500 AE \\ Enschede, The Netherlands
}

Received 6 December 1983, in final form 10 April 1984

\begin{abstract}
A new, sQuID-based, magnetometer has been developed for measurements of remanent and induced magnetisation. The advantage of this system compared to conventional SQLID-magnetometers is its horizontal access to the sensing coils. The access at room temperature is open at both ends. Main benefits of the horizontal access are a better accessibility to the space inside the sensing coils and the possibility for performing measurements on very long samples and materials which are transported through the access.

The prototype model that we constructed has a dipole sensitivity of $3 \times 10^{-11} \mathrm{Am}^{2} / \sqrt{\mathrm{Hz}}$ and a response time constant of $8 \mathrm{~ms}$. Design considerations and possibilities to improve the system are described.
\end{abstract}

\section{Introduction}

It is necessary for a multitude of applications that the magnetisation of a material is measured at room temperature. Depending on the type of application this magnetisation is present in remanent form or it has to be induced by means of an applied magnetic field. There are several techniques at one's disposal for performing these measurements (Zijlstra 1967, Bozorth and Dwight 1967, Foner 1981). When, however, extreme sensitivity is required SQUID-magnetometers are the most appropriate systems to be used (see e.g. Goree 1972, Sarwinski 1977 and Walton 1977).

SQUID-magnetometers can be divided into two types. One type is used to measure the remanent magnetisation of mainly geological samples and is therefore often called a rock magnetometer (Goree and Fuller 1976, Walton 1977, Barnard 1980), whereas the other type is used to investigate susceptibilities and hence called a susceptometer (Cukauskas et al 1974, Nave and Huray 1980, Vitale et al 1982). The main differences between the two types concern the maximum applicable magnetic field, the admissible sample diameter and the sample temperature range. Generally, rock magnetometers are not equipped with a magnet for the application of a static field. They have an access to the sensing coils of several centimetres in diameter and the sample is at room temperature. Susceptometers usually have a maximum applicable field of about 5 Tesla, their access diameter rarely exceeds one centimetre and measurements can be performed in a temperature range of $3-400 \mathrm{~K}$.

Maximum signal output is obtained in SQUID-magnetometers by placing the sample inside the sensing coils. For this purpose conventional magnetometers usually have a vertical access to the sensing coils, which is only open at the upper side (Cukauskas et al 1974, Barnard 1980, Nave and Huray 1980 , Vitale et al 1982). In only a few cases a bottom entry is used (Walton 1977, Barnard 1980). Compared to these configurations a horizontal access with open ends has several advantages (see $\S 2.1$ ). However, this type of entry is very rarely applied. As far as we know. only Goree has been working on a rock magnetometer with a horizontal access (Goree 1972, Goree and Fuller 1976). However, Goree (1976) mentions some important disadvantages of this system in relation to vertical access systems. It is expensive to construct and difficult and time consuming to repair or modify the sensor or shield assembly.

In this paper we present a sQuID-magnetometer with an open-ended horizontal access in which the problems mentioned above are eliminated. Compared to vertical access systems our magnetometer is certainly not more expensive to construct. Additionally, it can be dismantled in a very simple and rapid manner. Because we chose to design this magnetometer as a prototype to be used for various applications as a rock magnetometer as well as a susceptometer in different fields of research, it was not optimised for one specific application. Of course it is possible to improve the system for each particular application by appropriate modifications of the prototype model.

In $\S 2$ a number of important design considerations are reported, among which a qualitative comparison between a horizontal access system and conventional systems. A detailed description of our system is given in $\S 3$. The cryogenic part is described in $\S 3.1$ and the sensor assembly in $\S 3.2$. In $\S 3.3$ attention is paid to the sensitivity and dynamic behaviour of the system. Finally the versatility of the magnetometer is demonstrated in $\$ 3.4$ by some test experiments. A discussion of the features of our prototype follows in $\S 4$.

\section{Design considerations}

\subsection{Comparison with conventional systems}

Conventional vertical access systems can be represented by figure $1(a)$. It is essential that the sensor assembly (sensing-coil system and SQUID) is superconducting and for proper operation it therefore has to be cooled to a temperature below about $6 \mathrm{~K}$, usually realised by means of a liquid-helium bath cryostat. The magnet for the applied field can be a normal conducting coil outside the cryostat. It is, however, simpler and therefore more or less a practice to employ a superconducting magnet coil.
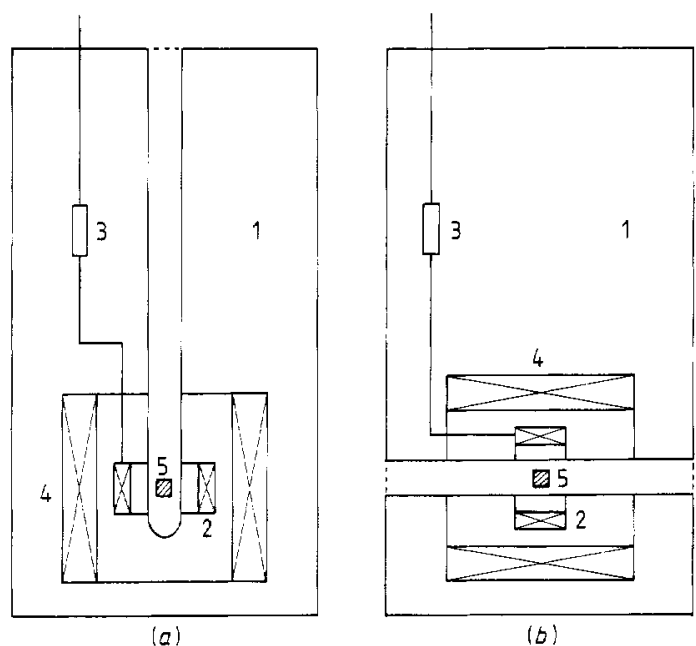

Figure 1. Schematic representation of conventional magnetometers: (a), system with vertical access; $(b)$, system with horizontal access. 1, cryostat; 2 , sensing-coil system; 3, SQUID; 4, magnet coil; 5 , sample. 
Conventional systems are used for measurements on samples which are small compared to the dimensions of the sensing-coil system. The sample is usually placed in a static position. A (harmonically) varying sample position is only used in a vibrating-sample magnetometer (Cerdonio et al 1977).

Our system is schematically given in figure $1(b)$. The remarks on the cryogenic requirements with regard to the superconducting components are valid for this magnetometer in the same way as for the vertical access systems. The horizontal access which is open at both ends exhibits a striking difference between the two systems. This access has some remarkable advantages:

(i) The open ends and the smaller length of the access result in a far better accessibility to the space inside the sensing coils. Therefore the sample handling is much simpler. It will also facilitate manipulation of a furnace when heating of the sample inside the access is desired.

(ii) It is now possible to measure the magnetisation of samples transported through the access. Therefore measurements on flowing gases or liquids can be performed. Furthermore, it is possible to measure the magnetisation of very long samples. This is a highly important advantage in the field of paleomagnetic applications (Collinson 1983).

\subsection{Specifications for the prototype}

In order to manufacture our prototype system simply and quickly, we decided to design an all-metal cryostat in which cooling is established by means of liquid helium. There are, however, two disadvantages with the use of metal components. Firstly, the inevitable Johnson noise will result in a loss of sensitivity and secondly, eddy currents will cause a deterioration of the dynamic behaviour.

Concerning the dipole sensitivity we were satisfied with a factor of 10 less than that of competitive commercial systems. Because these systems have a noise level of $(5-10) \times 10^{-12} \mathrm{Am}^{2}$ using an averaging time of 1 second (Collinson 1983), the noise level of our prototype had to be less than $10^{-10} \mathrm{Am}^{2} / \sqrt{\mathrm{Hz}}$. Again for the sake of simplicity in the prototype construction, we did not attempt to realise magnetic fields in the Tesla region but limited the required maximum field value to some $10 \mathrm{mT}$, corresponding to the maximum applicable field of some of the rock magnetometer systems. If the system is used as a susceptometer this will imply that the minimum detectable sample susceptibility is limited to $10^{-14} \mathrm{~m}^{3}$.

The cut-off for high-frequency signals due to the eddy currents has an especially disturbing effect on measurements of moving samples. It follows from calculations that in our design a time constant of $8 \mathrm{~ms}$ can be realised. In the first instance we were satisfied with this value as a maximum time constant for the prototype.

Another important specification of the system is the inner diameter of the access. The larger the diameter, the better the accessibility to the space inside the sensing coils will be, but it will be more difficult to shield the sensing coils properly from external magnetic noise (see $\$ 3.2$ ). We compromised with a diameter of $10 \mathrm{~mm}$.

Beside the sensitivity, the dynamic behaviour and the access diameter there were some more demands on the mechanical construction and the selection of materials. Firstly, it had to be simple to dismantle the system. Secondly, the temperature had to be fairly constant over the length of the access in order to perform accurate susceptibility measurements. The temperature variation should therefore be less than $1 \mathrm{~K}$. Finally, the time between helium refills had to be at least 3 days, so that the system could be continuously operational in spite of a weekend stop.

\section{Prototype}

\subsection{Cryogenics}

In our magnetometer the superconducting elements of the detection system are not cooled by direct contact with liquid helium, but instead they are rigidly mounted to the copper bottom plate of the helium reservoir (see figure 2). In this respect the accessibility of the detection elements is far better. Modification or repair can therefore be carried out much more simply. In this construction cooling is obtained by heat conduction to the helium bath. In order to optimise this conduction we used copper as intermediate material.

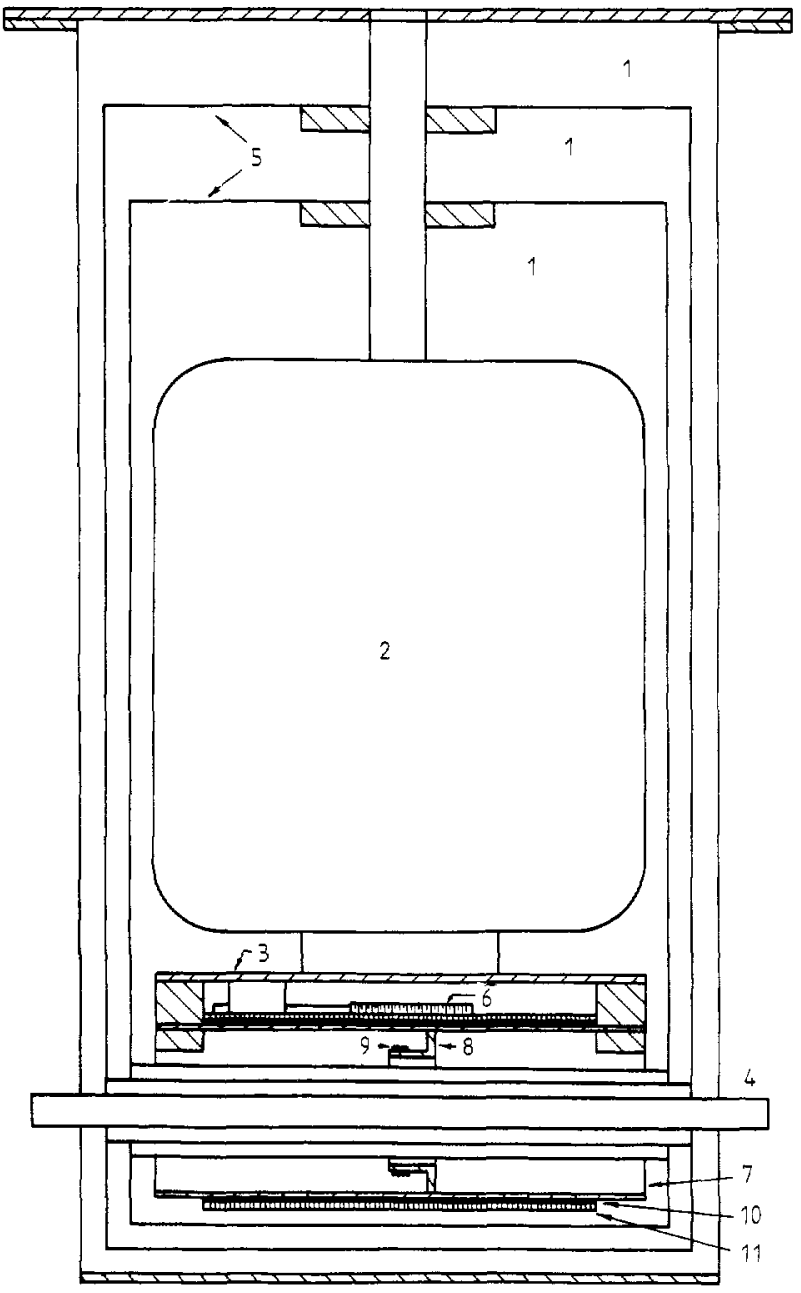

Figure 2. Schematic representation of the horizontal-access magnetometer; 1 , vacuum space; 2 , helium reservoir; 3 , bottom plate; 4 , access; 5 , radiation shields; 6 , sQuid (partly visible); 7 , copper cylinder; 8 , copper holder for pick-up coil; 9 , pick-up coil; 10, lead shield; 11, magnet coil.

Furthermore copper-to-copper contacts were provided with a thin gold layer. Most components of the sensor assembly are attached to a copper cylinder which is fastened to the bottom plate. Only the SQUID is placed in a separate holder. The insulation of the helium bath is established by a vacuum space with two vapour-cooled radiation shields. The radiation shields are also placed concentrically around the pipe of the horizontal access. The cryostat system is $780 \mathrm{~mm}$ high and measures $360 \mathrm{~mm}$ in diameter. 
The whole system, helium reservoir, radiation shields and outer cryostat shell, is suspended from the top plate of the cryostat. To dismantle the system the access pipe has to be first removed, and then the outer shell can be lowered. The dismantling of the radiation shields is carried out in the same way, first the pipe and then the part of the shield around the helium reservoir. Following this procedure the system can be dismantled in less than one hour.

As mentioned in $\S 2.2$ the temperature of the sample material is of crucial importance for susceptibility measurements. In order to minimise the temperature gradient along the access pipe, we used a copper tube having a wall thickness of $1 \mathrm{~mm}$ and, as mentioned above, an inner access diameter of $10 \mathrm{~mm}$. It appeared from measurements that the difference between room temperature and the temperature at the centre of the access is $1.5 \mathrm{~K}$. Improvement of the heat contact between the access ends and the outer shell of the cryostat, which are separated by means of rubber $O$ rings, will result in a further reduction of this temperature difference to $0.4 \mathrm{~K}$.

In operation the SQUID holder is cooled to $5 \mathrm{~K}$ and the temperatures of the radiation shields are about 180 and $80 \mathrm{~K}$. In this situation the helium evaporation is 0.18 litres of liquid helium per hour. With a reservoir volume of 18 litres this results in an operating time of four days.

\subsection{Sensor assembly}

In order to pick up the signal applied in the access, we use one pick-up coil directly connected to the SQUID. Noise reduction from the surroundings is achieved by means of a cylindrical lead shield, on which we wound the magnet coil.

3.2.1. Pick-up system. In order to obtain the maximum signal from a material in the access, the diameter of the pick-up coil has to be as small as possible. There is, however, a practical limit to this diameter because it is necessary that the pipes of the radiation shields have a certain thickness for proper thermal conduction, whereas the distance between successive pipes can not be made too small in order to avoid contact due to thermal shrinkage. In our prototype we chose $4 \mathrm{~mm}$ for this distance and 0.5 to $1.0 \mathrm{~mm}$ for the thickness of the pipes, resulting in a pickup coil diameter of $40 \mathrm{~mm}$. The pick-up coil and the connecting leads to the SQUID were made of NbTi single-core wire (diameter $0.15 \mathrm{~mm}$, without copper stabilisation). The number of turns of the pick-up coil has to be so chosen that its self-inductance matches the effective self-inductance of the signal coil in the SQUID. In our system we use a commercially available RF-SQCID system (SHE-330 with TSQ-sensor). Because the effective signal coil self-inductance is $2 \mu \mathrm{H}$ optimum signal transfer from pickup coil to SQUID is realised by four pick-up turns. The resulting overall flux sensitivity from pick-up coil to output of the SQLID system is measured as $(4.30 \pm 0.03) \times 10^{10} \mathrm{~V} \mathrm{~Wb}^{-1}$ (SHE 330 in sensitivity mode $\times 1$ ).

As mentioned above the noise from the surroundings is suppressed by means of a lead cylinder. This cylinder is made of thin lead foil $(0.12 \mathrm{~mm}$ thick) wrapped once around the copper cylinder and soldered with Woods metal. The shielding effect of the cylinder is determined by the ratio of its length and diameter. Our cylinder (length $200 \mathrm{~mm}$, diameter $67 \mathrm{~mm}$ ) provides a noise flux reduction by a factor of $1.1 \times 10^{-5}$ in the centre for homogeneous disturbing fields (Thomasson and Ginsberg 1976). The calculated effective noise reduction in the total pick-up area, in this case, amounts to $7.2 \times 10^{-6}$.

3.2.2. Applied magnetic field. In order to apply a magnetic field in the access we wound a single-layer coil around the lead shield. The coil consists of about 900 turns of NbTi multi-filament wire with copper stabilisation $(\varnothing 0.23 \mathrm{~mm})$. Heating the lead shield above its critical temperature $(7.2 \mathrm{~K})$, and then cooling it down again, enables us to trap an applied magnetic field in the superconducting lead cylinder. In this procedure the cylinder is only heated along one line over its total length. This local heating involves much less dissipation than if the whole lead cylinder were heated, and therefore a considerable reduction of the extra helium evaporation is obtained (difference in the order of $90 \%$ ). Furthermore, it results in a fast field-trapping procedure. The applied field enters the interior of the cylinder after heating for less than a second.

The attained field in the centre for a certain current through the coil is given by $5.190 \mathrm{mT} \mathrm{A}^{-1}$ (absolute accuracy $0.01 \mathrm{mT}$ ). This linear relation between field and current is valid for fields up to about $9 \mathrm{mT}$. Larger fields up to $12 \mathrm{mT}$ are possible but then it is not possible to properly reproduce trapping of the field. Although the required $10 \mathrm{mT}$ is thus realised the maximum field value is still somewhat low because the critical field of lead at, for instance, $5 \mathrm{~K}$ is about $40 \mathrm{mT}$. This discrepancy is caused by the influence of surface effects in the lead (surface strains, chemical purity etc, Schweitzer (1968)) and by end effects in the lead cylinder (Coffey et al 1963).

The homogeneity of the applied field for different field values is given in figure 3 . The homogeneity for trapped fields smaller than $9 \mathrm{mT}$ is exactly the same as that for the single-layer coil. Apparently the lead cylinder does not act like a type I superconductor, otherwise we should have obtained curve $\mathrm{C}$ in figure 3, which is calculated from the results of Bardotti et al (1964). This is in good agreement with the expected so-called very incomplete Meissner effect measured on lead cylinders by Rabinowitz et al (1973). When the applied field exceeds $9 \mathrm{mT}$,

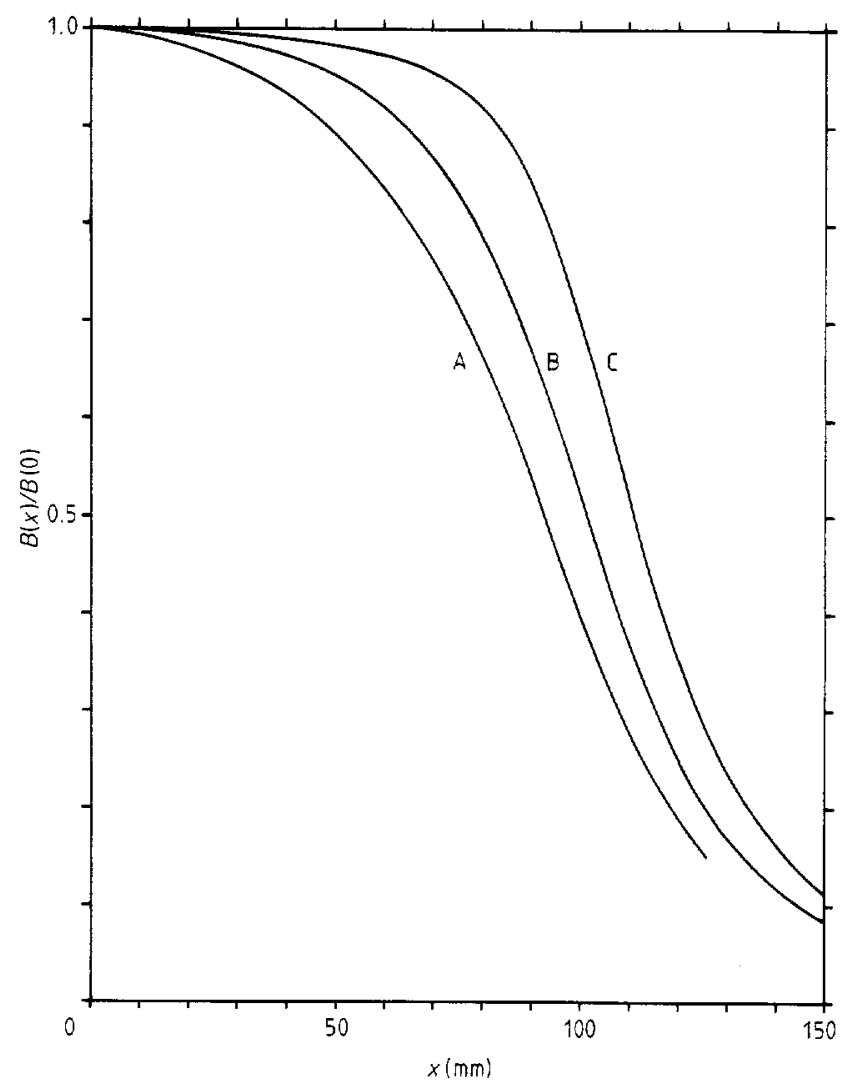

Figure 3. Homogeneity of the applied field on the axis (centre at $x=0$ ). A, applied field $11.7 \mathrm{mT} ; \mathrm{B}$, applied field smaller than $9 \mathrm{mT}$ (equal to the field of the single-layer coil); $\mathrm{C}$, calculated field in case the cylinder is a type I superconductor. 
the homogeneity (curve A) is worse because of the cylinder-end effects previously mentioned (Coffey et al 1963).

\subsection{Sensitivity and dynamic behaviour}

3.3.1. Sensitivity. The sensitivity of the magnetometer is determined by the noise level of the system itself and by that of the ambient laboratory magnetic field. The latter is suppressed by approximately a factor of $10^{5}$ by the lead shield.

The noise level of the system is mainly determined by the thermal noise of the copper elements. Consider a conducting cylinder with length $l$, radius $R_{\mathrm{c}}$ and thickness $\delta$ inside the pickup coil. Based on considerations of Bakker and Heller (1939) the noise from the cylinder picked up by the pick-up coil can be written as

$$
\overline{\varphi_{\mathrm{N}}^{2}}=\frac{4 \sigma_{0} k T \Delta \nu \delta}{2 \pi R_{0}} \int_{-1 / 2}^{1 / 2} M^{2}(z) \mathrm{d} z
$$

with $\sigma_{0}$ the conductivity of the cylinder material, $T$ its temperature and $\Delta \nu$ the frequency bandwidth. $M(z)$ is the mutual inductance between the pick-up coil and a ring element of the cylinder at a distance $z$ from the pick-up coil. Equation (1) is only valid in the low-frequency region because for higher frequencies the reduction of the noise flux due to eddy currents must be taken into account. Based on the work of Clark et al (1970) we approximated the electrical resistivity $\left(=1 / \sigma_{0}\right)$ of copper as $5 \times 10^{-10} \Omega \mathrm{m}$ at temperatures below $20 \mathrm{~K}$ increasing to $1.7 \times 10^{-8} \Omega \mathrm{m}$ at $300 \mathrm{~K}$. Using equation (1) we calculated the contribution of the various elements expressed in the noise in the pick-up coil, the results being listed in table 1 . The intrinsic SQUID noise, equal to $0.04 \varphi_{0} / \sqrt{\mathrm{Hz}}$, is a factor 35 less than the total noise of the system.

The RMS noise can be reasonably well approximated from measurements by taking the maximum peak-to-peak noise signal over a 100 second interval multiplied by 0.2 . A noise recording of our magnetometer is presented in figure 4 and as can be determined the noise level is about $0.15 \mathrm{mV} / \sqrt{\mathrm{Hz}}$. This results with the overall flux sensitivity stated in $\S 3.2$ in a pick-up flux sensitivity of $1.7 \varphi_{0} / \sqrt{\mathrm{Hz}}$. This value is in very good agreement with the calculated noise flux in table 1 and therefore it can be concluded that the noise from the laboratory environment is negligible. Using the shielding factor of $10^{5}$ we calculated that the noise level of the ambient laboratory field is less than $0.1 \mu \mathrm{T}$.

The dipole sensitivity can be derived from the pick-up flux sensitivity. The flux picked up from a point dipole $m$ by a pickup coil of $N$ windings with radius $R_{\mathrm{pu}}$ is given by

$$
\varphi_{\mathrm{pu}}=\frac{\mu_{0} N m}{2 R_{\mathrm{pu}}} \text {. }
$$

Table 1. Calculated influence of the copper system elements on

\begin{tabular}{|c|c|c|c|}
\hline System element & $\begin{array}{l}\text { Noise flux in } \\
\text { the pick-up } \\
\text { coil } \\
\left(\varphi_{0} \mathrm{~Hz}^{-1 / 2}\right)\end{array}$ & $\begin{array}{l}\text { Time } \mathrm{c} \\
\tau \\
(\mathrm{ms})\end{array}$ & $\begin{array}{l}\text { nstant } \\
\nu_{\mathrm{c}} \\
(\mathrm{Hz})\end{array}$ \\
\hline Holder for pick-up coil & 0.70 & 7.01 & 22.7 \\
\hline Cylinder & 0.51 & 36.3 & 4.38 \\
\hline Outer radiation shield & 0.44 & 0.74 & 216 \\
\hline Inner radiation shield & 1.04 & 4.40 & 36.4 \\
\hline Access pipe & 0.15 & 0.20 & 796 \\
\hline Subtotal & 1.43 & & \\
\hline SQUID intrinsic noise & 0.04 & & \\
\hline Total & 1.43 & & \\
\hline
\end{tabular}
noise and dynamic behaviour $\left(\varphi_{0}=2.07 \times 10^{-15} \mathrm{~Wb}\right)$.
Because in our system $N=4$ and $R_{\mathrm{pu}}=20 \mathrm{~mm}$ the minimum dipole moment that can be detected amounts to $2.8 \times$ $10^{-11} \mathrm{Am}^{2} / \sqrt{\mathrm{Hz}}$. This value is well below the limit of $10^{-10} \mathrm{Am}^{2} / \sqrt{\mathrm{Hz}}$ posed in $\S 2.2$. As an illustration, the output of the system for an input change corresponding to a point dipole of $8.6 \times 10^{-10} \mathrm{Am}^{2}$ is given in figure 4. This point dipole is simulated by means of a small coil inside the access.

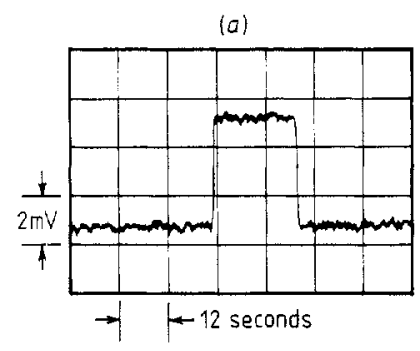

(b)

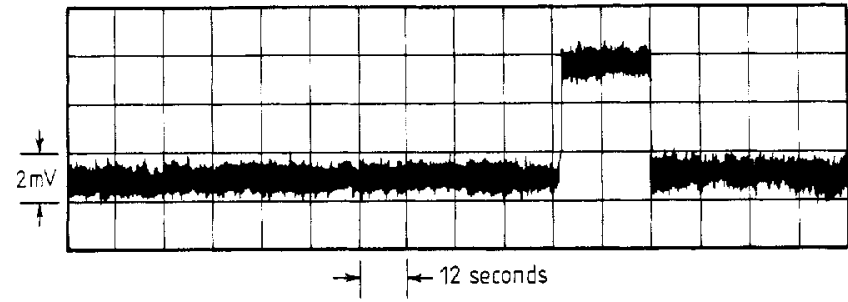

Figure 4. System output noise recording and the response to an input change of $8.6 \times 10^{-10} \mathrm{Am}^{2}$ (no field applied). (a), low pass $1 \mathrm{~Hz}$; (b), low pass $10 \mathrm{~Hz}$.

If a magnetic field is applied the noise is somewhat larger especially for higher frequencies. For instance, with a field of $5 \mathrm{mT}$ the noise level is $0.2 \mathrm{mV}$ for the frequency range $0-1 \mathrm{~Hz}$ and $4.0 \mathrm{mV}$ for $0-10 \mathrm{~Hz}$. This rise in noise is caused by mechanical vibrations of metal system elements which are magnetised by the applied field.

3.3.2. Dynamic behaviour. As mentioned in $\S 2.2$ our system will show a low-pass frequency response due to eddy currents in the metal components. A rough indication of the effect of the various components can be obtained from their time constants $L / R, L$ being the self inductance of the component and $R$ its resistance. Because all significant components are cylinders inside or around the pick-up coil, the resistance must be considered with respect to a current in the tangential direction. We calculated the time constants of the various components using the conductivity of copper as mentioned before and the self-inductance as can be evaluated according to Grover (1962). The resulting time constants and corresponding characteristic frequencies are listed in table 1.

The holder for the pick-up coil will have a dominant effect because its time constant is low and more important because it is closely coupled to the pick-up coil. Also the copper cylinder and the inner radiation shield will affect the frequency response, but these will only have a minor influence because the inductive coupling to the pick-up coil is much weaker. The dynamic behaviour of the system is thus largely characterised by a time constant of $7 \mathrm{~ms}$, which implies a $-3 \mathrm{~dB}$ point at about $23 \mathrm{~Hz}$.

In order to measure the dynamic behaviour of our magnetometer we used a small input coil in the access. The coil had eight windings and a diameter of $2.2 \mathrm{~mm}$. The results of the measurements are given in figure 5 . We can conclude that the dynamic behaviour of our magnetometer can be characterised 


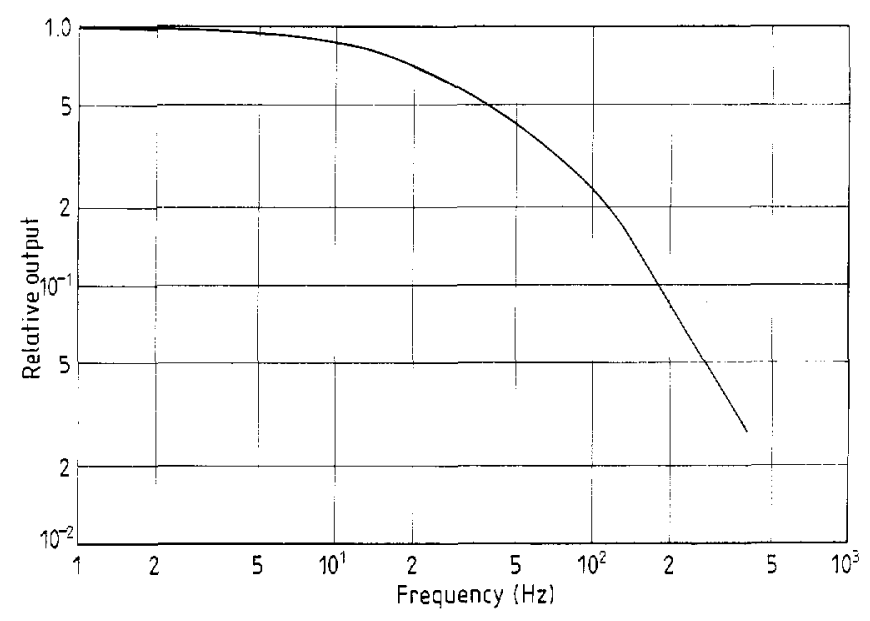

Figure 5. Dynamic behaviour: relative system output due to an AC current in the input coil.

by a low-pass frequency response having a $-3 \mathrm{~dB}$ point at $20 \mathrm{~Hz}$, corresponding to a system response time constant of $8 \mathrm{~ms}$, which comes close to what may be expected according to the rough approximation mentioned above.

3.3.3. Possible improvements of the sensitivity and dynamic behaviour. Because both the sensitivity and dynamic behaviour were quite satisfactory for our prototype, we have so far not tried to improve them. However, it is clear that, if necessary, important improvements can be achieved by reducing the influence of the copper system elements. Table 1 shows which components are mainly responsible for the noise level as well as the dynamic behaviour of the system. It indicates, for instance, that it is far better to reduce the influence of the inner radiation shield or the holder of the pick-up coil than to eliminate the effect of the access pipe.

The influence of these system elements can be diminished by using copper wires or strips instead of bulk copper. In principal, it is only necessary in the case of the access pipe to embed these strips or wires in an insulating material because only the access pipe must be vacuum tight. This will be more complex to realise and, as can be seen from table 1 , it is only reasonable if ultimate sensitivity is desired. In this limit the sensitivity is determined by the intrinsic SQUID noise and, according to the above calculations, this results in a dipole sensitivity of less than $10^{-12} \mathrm{Am}^{2} / \sqrt{\mathrm{Hz}}$.

Another improvement which will be profitable for susceptibility measurements is the enlargement of the maximum value of the applied magnetic field. Because in our prototype the maximum field value is limited by the critical magnetic field of the material of the superconducting cylinder, this enlargement can, for instance, be realised by using niobium instead of lead. The resulting gain in sensitivity may be somewhat reduced by an increase in noise due to mechanical vibrations in the system. Therefore, in order to obtain the optimum benefit of higher applied fields, these vibrations will have to be limited.

\subsection{Test experiments}

As previously indicated the possible applications of our magnetometer system can be divided into two groups. On the one hand the instrument can be used to measure remanent magnetisation and on the other susceptibility by applying a magnetic field. We performed some test experiments, which will be discussed in the following sections.
3.4.1. Remanent magnetisation. The main application in this field is measurements on rock samples for paleomagnetic studies. We received some rock samples from the Paleomagnetic Laboratory of the University of Utrecht in order to test our system. With regard to the sensitivity as well as the ease of sample handling the test experiments were quite satisfactory. Because the prototype was not designed as a typical rock magnetometer, two disturbing effects hamper correct measurements on the remanent magnetisation of the samples. Firstly, the system is not equipped with mu-metal shields for the creation of a field-free space, and therefore the earth's magnetic field is trapped in the lead cylinder when the system is cooled down. This results in an induced magnetisation of the rock sample which adds to the natural remanent magnetisation. Secondly, the length of the pick-up coil is small compared to that of the rock sample (10 to $15 \mathrm{~mm}$ ) so that the measured magnetisation is somewhat smaller than the real mean value. It is of course possible to solve this problem simply by using a pick-up coil system in a Helmholtz configuration.

An important benefit of the horizontal access is the possibility of performing measurements on long samples. As a simulation of this application we measured the induced magnetisation of a rod of perspex (diamagnetic) having a length of about $0.5 \mathrm{~m}$ and some $5 \mathrm{~mm}$ in diameter. The applied magnetic field was roughly $5 \mathrm{mT}$. We transported the rod with a constant velocity through the access and the signal output of the system is presented as a function of the time in figure 6 . This plot can be considered as the magnetisation as a function of the position along the rod. The variation of the signal is caused by inhomogeneities of the rod (e.g. magnetic impurities, diameter variations).

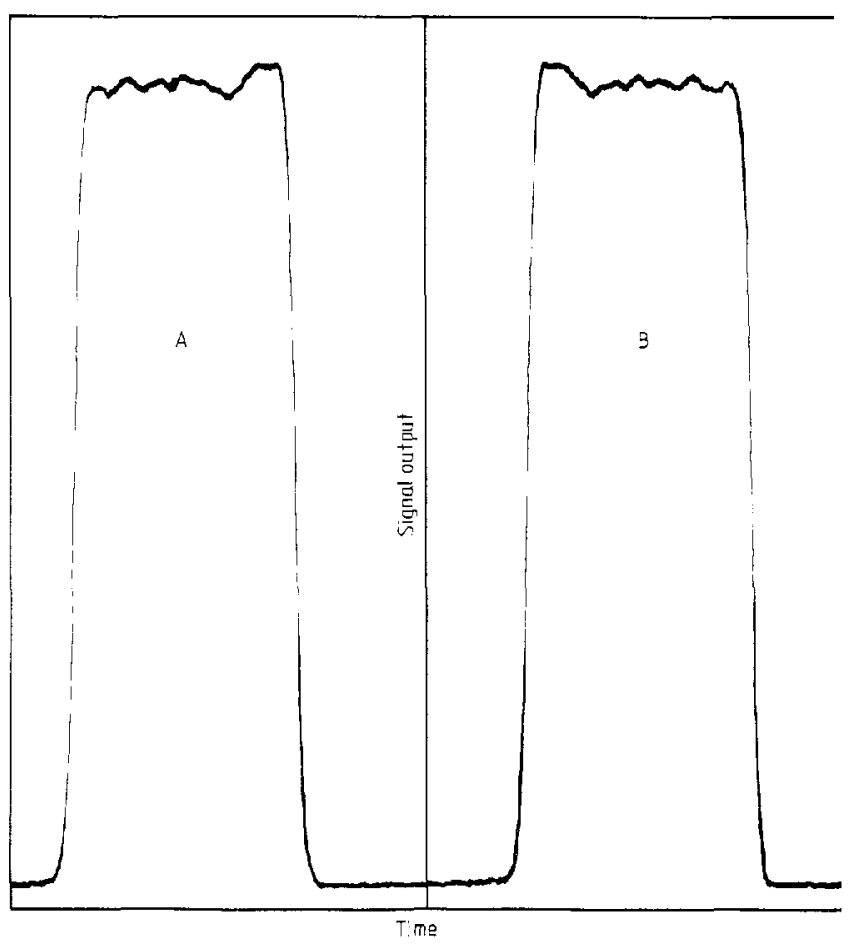

Figure 6. System output due to the transportation of a perspex rod through the access; $A$ and $B$ corresponding to opposite directions.

3.4.2. Susceptibility. The susceptibility $\chi$ of a material can be accurately measured by placing a small volume $V$ in the centre 


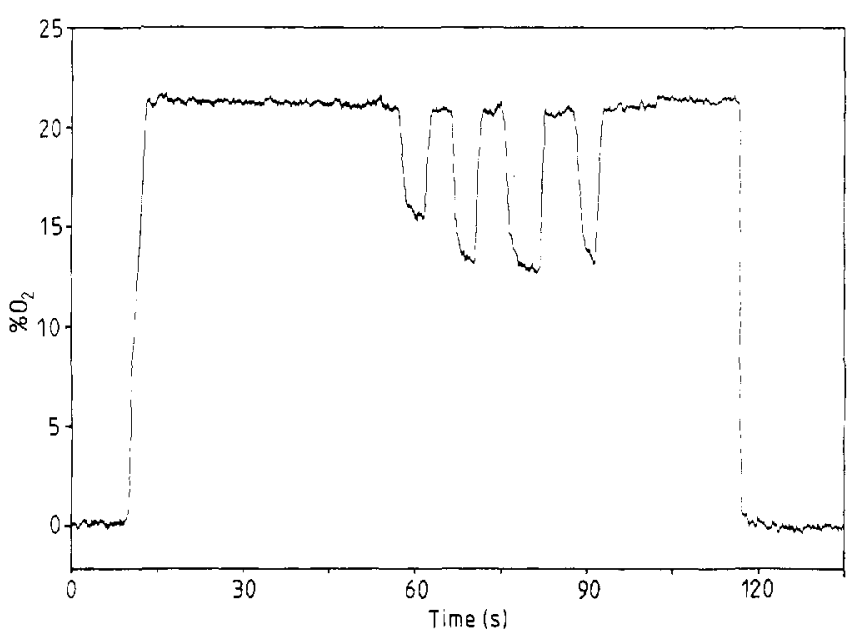

Figure 7. Recording of the oxygen concentration in the access in expired air.

of the pick-up coil when a magnetic field $H$ is applied. The output of the system caused by this sample can be calculated using the dipole approximation in equation (2) $\left(m=\chi^{V H}\right)$ and the overall flux sensitivity. The resulting signal is then $(5.38 \pm 0.05) \times 10^{6} \mathrm{~V} \mathrm{~A}^{-1} \mathrm{~m}^{-2}$. It is simple to evaluate the susceptibility of a material from this overall dipole sensitivity if the volume and the applied field are known.

We performed a calibration of the system using $60 \mathrm{mg}$ of $\mathrm{Gd}_{2} \mathrm{O}_{3} \quad\left(\chi=13.660 \times 10^{-3}\right.$ at $\left.293 \mathrm{~K}\right)$ in a magnetic field of $6 \mathrm{mT}$. The signal transfer is thus measured as $(5.33 \pm 0.06) \times 10^{6} \mathrm{~V} \mathrm{~A}^{-1} \mathrm{~m}^{-2}$, being in very good agreement with the overall dipole sensitivity calculated above.

Measurement of the susceptibility of course makes it possible to determine the concentration of a material with relatively high susceptibility in a background material with relatively low susceptibility. One of the applications is the detection of oxygen in air. Oxygen is the only paramagnetic component in air, besides $\mathrm{NO}$ and $\mathrm{NO}_{2}$ that are present in negligible concentrations. As a further demonstration of the versatility of the system, we present some measurements on oxygen concentration in expired air. The zero-level in these measurements is fixed by blowing nitrogen gas through the access, while a calibration is simply carried out by using $100 \%$ oxygen. A plot of four expirations through the access is shown in figure 7 . As a possible medical application these registrations can be used to obtain information about the lung function (Kreuzer and Kimmich 1974).

\section{Discussion}

We have developed a SQuID-magnetometer with an open-ended horizontal room-temperature access in a cryostat which can be easily dismantled. First test experiments were very promising and showed the system's versatility for measurements on remanent and induced magnetisation. Contacts with industry and scientific research have resulted in a growing number of applications in this field. We are now optimising the system for use as rock magnetometer and as susceptometer. Both types require some specific modifications of the prototype model.

In the case of the rock magnetometer application, the dipole sensitivity has to be improved by at least a factor of 3 . Further, the system has to be equipped with a pick-up system capable of sensing in three perpendicular directions in order to derive the magnetisation as a vector in space. Other requirements for a rock magnetometer are a field-free space inside the sensing coil system and a relatively large region with uniform dipole sensitivity. Finally the access diameter must be enlarged up to several centimetres.

The susceptometer application also requires an improvement of the dipole sensitivity. Furthermore the maximum applicable field has to be increased from $10 \mathrm{mT}$ to some $5 \mathrm{~T}$. In order to realise the improvement of the dipole sensitivity, also in the case of higher applied fields, a reduction of mechanical vibrations in the system is necessary.

\section{References}

Bakker C J and Heller G 1939 On the Brownian motion in electric resistances

Physica 6 262-74

Bardotti G, Bertotti B and Gianolio L 1964 Magnetic configuration of a cylinder with infinite conductivity J. Math. Phys. 5 1387-90

Barnard B R 1980 The design of dewar systems for geophysical SQUID magnetometers

Proc. IInd Int. Conf. Superc. Quant. Devices (West Berlin: De Gruyter) pp 633-50

Bozorth R M and Dwight K 1967 Magnetic measurements: Experimental techniques with general applicability for the study of magnetic phenomena

J. Appl. Phys. 38 1505-9

Cerdonio M, Mogno F, Romani G L, Messana C and Gramaccioni C 1977 Oscillating sample superconductin: magnetometer

Rev. Sci. Instrum. 48 300-6

Clark A F, Childs G E and Wallace G H 1970 Electrical resistivity of some engineering alloys at low temperatures Cryogenics 10 295-305

Coffey D L, Gauster W F and Rorschach H E Jr 1963

Fluxtrapping in hollow soft superconducting cylinders Appl. Phys. Lett. 3 75-6

Collinson D W 1983 Methods in rock magnetism and paleomagnetism. Techniques and instrumentation (London: Chapman and Hall)

Cukauskas E J, Vincent D A and Deaver B S Jr 1974 Magnetic susceptibility measurements using a superconducting magnetometer

Rev. Sci. Instrum. 45 1-6

Foner S 1981 Review of magnetometry

IEEE T-MAG $173358-63$

Goree W S 1972 Advances in superconducting instrument systems

Proc. Appl. Superc. Conf., Annapolis (New York: Academic) pp 640-8

Goree W S and Fuller M 1976 Magnetometers using RF-driven SQUID's and their applications in rock magnetism and paleomagnetism

Rev. Geophys. Space Phys. 14 591-608

Grover F W 1962 Inductance calculations, working formulas and tables (New York: Dover)

Kreuzer F and Kimmich H P 1974 Recent developments in oxygen polarography as applied to physiology Proc. Interdisc. Symp. Measurement of Oxygen, Odense (Amsterdam: Elsevier) pp 123-35

Nave S E and Huray P G 1980 Micromagnetic susceptometer Rev. Sci. Instrum. 51 591-6 
Rabinowitz M, Garwin E L and Frankel D J 1973 An investigation of the very incomplete Meissner-effect Lett. Nuovo Cim. 7 1-4

Sarwinski R E 1977 Superconducting instruments Cryogenics 17 671-9

Schweitzer D G 1968 Hysteresis in superconductors III. Effect of surface energies on the shielding and field-retaining properties of hollow cylinders of type-I superconductors

Phys. Rev. 173 461-72

Thomasson J W and Ginsberg D M 1976 Magnetic field shielding by a superconducting cylindrical tube of finite length Rev. Sci. Instrum, $47387-8$

Vitale S, Morante S and Cerdonio M 1982 Superconducting susceptometer for high-accuracy routine operation Rev. Sci. Instrum. 53 1123-8

Walton D 1977 Archaeomagnetic intensity measurements using a SQUID magnetometer

Archaeometry 19 192-200

Zijlstra H 1967 Experimental methods in magnetism vol. 2

(Amsterdam: North-Holland) 